\title{
Prospective comparative multicenter study of suctioning MPCNL with the aid of a patented system, traditional MPCNL and ureteroscopy in treating impacted upper ureteral stones
}

\author{
Zhongsheng Yang ${ }^{1 \#}$, Chunxiang Luo ${ }^{6 \#}$, Donghua Xie ${ }^{7,8 \#}$, Xiaohui Liao ${ }^{5 \#}$, Xuanxi Qiu ${ }^{1}$, Xiaolin Deng ${ }^{1}$, Lunfeng Zhu ${ }^{1}$, Difu Fan ${ }^{1}, Z^{2}$ uofeng \\ Peng ${ }^{1}$, Wen Qin ${ }^{1}$, Qigui Liu ${ }^{2}$, Tiejun Pan ${ }^{3}$, Zhiqiang Chen ${ }^{4}$, Zhangqun Ye ${ }^{4}$ and Leming Song ${ }^{1 *}$ \\ ${ }^{1}$ Department of Urology, The Affiliated Ganzhou Hospital of Nanchang University, Ganzhou, Jiangxi 341000, China \\ ${ }^{2}$ Department of Urology, Kunming General Hospital of Chengdu Military Region, Kunming, Yunnan 650032, China \\ ${ }^{3}$ Department of Urology, Wuhan General Hospital of Guangzhou Military Region, Wuhan, Hubei 430070, China \\ ${ }^{4}$ Department of Urology, Tongji Hospital, Tongji Medical College, Huazhong University of Science and Technology, Wuhan, Hubei 430030, China \\ ${ }^{5}$ Dermatology Institute of Gan County, Jiangxi, China \\ ${ }^{6}$ Fushan Branch, Liaobu Township Community Health Service Center, Dongguan, Guandong 523430, China \\ ${ }^{7}$ Nova Southeastern University, Fort Lauderdale, FL 33314, U.S.A \\ ${ }^{8}$ Urological Research Network, Hialeah, FL 33016, U.S.A
}

\begin{abstract}
Objective: To determine an efficient method for treating upper ureteral impacted stones, we compared the outcome of minimally invasive percutaneous nephrolithotomy (MPCNL) with the aid of our patented system (PMPCNL), traditional MPCNL, and transurethral ureteroscopy. PATIENTS AND Methods: From September 2009 to Septmber 2014, 4 hospitals in China had executed this prospective multicenter study. A total of 273 patients with complicated impacted upper ureteral stones above the level of L4 were randomly divided into 3 groups. Group 1 included 91 patients who were treated with MPCNL with the aid of a patented system. Group 2 included 91 patients who were treated with traditional MPCNL .Group3 included 91 patients who were treated with ureteroscopy. The patients underwent postoperative shock wave lithotripsy, when necessary. The operative time, stone clearance rate, operative complication markers (amount of intraoperative bleeding and postoperative fever rate), and cost of treatment were compared.
\end{abstract}

Results: A significantly shorter operative time, greater rate of stone clearance, lower need for postoperative shock wave lithotripsy, and lower rate of postoperative fever was found in group 1 and group 2 compared to group $3(p<0.05)$. However, the cost of treatment and amount of intraoperative bleeding in groups 1 and 1 were significantly greater. PMPCNL group was superior to MPCNL group in terms of operation time, incidence of postoperative fever, and stone-free rate by one surgery.

Conclusion: MPCNL with the aid of the patented system could be the first choice in treating complicated impacted upper ureteral stones above the level of L4.

\begin{abstract}
Abbreviations: URS: Ureteroscopy; PCNL: percutaneous nephrolithotomy; PMPCNL: patented system assisted minimally invasive nephrolithotomy; MPCNL: minimally invasive nephrolithotomy; IVU: Intravenous urography; CT: Computerized tomography; KUB: X-ray of kidneys, ureters, and bladder; UTI: Urinary tract infection; SWL: Shock wave lithotripsy
\end{abstract}

\section{Introduction}

Impacted ureteral stones usually refer to ureteral stones located in a same place for more than 2 months, or stones that prevent the passage of a guidewire or a ureteral catheter $[1,2]$. In the southern part of China, impacted upper ureteral stones are very common, resulting from their being surrounded by ureteral polyps, or from reduced cavitation effect due to a lack of aqueous medium on the surface of the stone [3]. The outcome of shock wave lithotripsy (SWL) in impacted upper ureteral stones was not ideal and nowadays ureteroscopy (URS) and percutaneous nephrolithotomy (PCNL) have become the main modalities. In order to increase the efficacy of stone clearance, we invented a patented system (patent number ZL200820137434.6) with suctioning ability to facilitate minimally invasive PCNL (PMPCNL). This system was found to be very effective in clearing kidney stones in a previous study we conducted, compared to standard tract PCNL [4]. We designed this current study to compare the outcome of PMPCNL versus traditional minimally invasive PCNL (MPCNL) versus URS, sought to find an effective and safe method in treating the impacted upper ureteral stones.

Correspondence to: Leming Song, MD, Department of Urology Ganzhou people’s hospital, 17 Hongqi Avenue, Ganzhou Jiangxi 341000, China, Tel: (01186) -797-8120226, Fax: (011-86) -797-8112320, Email: xdhmd66@hotmail.com \#contribute equally

${ }^{*}$ Corresponding author

Received: January 05, 2017; Accepted: January 27, 2017; Published: January 30, 2017 


\section{Patients and methods}

\section{Clinical data}

From September 2009 to Septmber 2014, 4 hospitals in China had executed this prospective multicenter study with a total of 273 patients including 157 males and 116 females. Their age ranged from 19 to 75 years. There were 126 patients with left ureteral stone and 147 patients with right ureteral stone. All patients had B ultrasonography of the urinary system, intravenous urography (IVU), or CT of the urinary system. All these patients had a ureteral stone at the proximal segment of the ureter above the level of L4. These stones were diagnosed as impacted ureteral stones either because the stone was found to have been in the same position for more than 2 months, or an IVU contrast agent could not pass the stone with at least a moderate degree of hydronephrosis with ectasis of the renal pelvis more than $4 \mathrm{~cm}$. The patients were fully instructed on the nature of the study, and informed consent was obtained. Our exclusion criteria included coagulopathy, serious heart disease or pulmonary insufficiency, severe kyphosis and scoliosis deformity, extreme obesity, active infection, urinary tract abnormalities, a simultaneous kidney stone needing operation, and pregnancy. We used a random number table to divide the patients into 3 groups with 91 patients in each group, based on the admission sequence of the patients. Group 1 received ureteroscopy (URS group); Group 2 received traditional MPCNL (MPCNL group); Group 3 received PMPCNL (PMPCL group). In Group 1, the stone size ranged from $7 \mathrm{~mm} \times 5 \mathrm{~mm}$ to $27 \mathrm{~mm} \times 15 \mathrm{~mm}$, the average size was $15 \mathrm{~mm} \times 8.5$ $\mathrm{mm}$. There were 64 patients in this group with moderate hydronephrosis and 27 patients with severe hydronephrosis. There were 13 patients complicated by urinary tract infection (UTI) and 9 patients who had failed SWL therapy. The stone size in Group 2 ranged from $7 \mathrm{~mm} \times 6 \mathrm{~mm}$ to $26 \mathrm{~mm} \times 16 \mathrm{~mm}$ with an average size of $16 \mathrm{~mm} \times 8.5 \mathrm{~mm}$. There were 60 patients in this group with moderate hydronephrosis and 31 patients with severe hydronephrosis. There were 13 patients complicated by UTI and 6 patients who had failed shock wave lithotripsy (SWL) therapy. In Group 3, the stone size ranged from $8 \mathrm{~mm} \times 5 \mathrm{~mm}$ to $29 \mathrm{~mm} \times 15$ $\mathrm{mm}$, the average size was $17 \mathrm{~mm} \times 9 \mathrm{~mm}$. There were 65 patients in this group with moderate hydronephrosis and 26 patients with severe hydronephrosis. There were 15 patients complicated by urinary tract infection (UTI) and 7 patients who had failed SWL therapy. There were no statistically significant differences in general data between the 3 groups including sex, age, stone size, severity of hydronephrosis, and rate of complications (Table 1).

\section{Treatment methods}

Surgery took place under continued epidural anesthesia or general anesthesia. Group 1 received transurethral ureteroscopy using a Holmium laser. A Wolf $\mathrm{F}_{8 / 9.8}$ rigid ureteroscope was inserted into the ureter with stone, the stone was then broken using Holmium laser into gravels less than $4 \mathrm{~mm}$ in size. All patients were examined for potential residual stones by KUB and B ultrasound 3 days postoperatively. For those stone gravels refluxed to the kidney by a saline infusion and lithotripsy that had a size greater than $4 \mathrm{~mm}$, they were treated with SWL 3-7 days postoperatively.

Group 2 received traditional MPCNL. The patient was first placed in a lithotomic position and then a prone position. The abdomen was not boosted, as previously described $[4,5]$. Ultrasonography-guided percutaneous punctures were made with an 18-gauge coaxial needle into the targeted calix (middle/upper kidney calices). The puncture point was in the 11 st or 12 th rib infracostal margin, between the posterior axillary line and scapula line. Zebra guidewire was inserted and fixed. The puncture needle was then taken out. After a $0.5-0.7 \mathrm{~cm}$ skin incision, the dilatation of the percutaneous tract was performed serially over the guidewire with a fascial dilator to $16 \mathrm{~F}$. A16Fr peelaway sheath was placed at the percutaneous access port. Subsequently, a small diameter nephroscope $(12.3 \mathrm{~F})$ was inserted through the sheath to observe stones. Infusion of normal saline using an infusion pump was carried out to maintain clear surgical field. Holmium laser (3.5 4.0 Jx15HZ) lithotripsy was then performed after finding the stone in the upper ureter. Gravels were flushed out by hydraulic pressure from the perfusion pump, or extracted by forceps manually. After examination to find whether there were any residual stones inside the upper ureter, the renal pelvis, and the renal calyces, we antegradely indwelled a $6 \mathrm{~F}$ double-J pigtail stent from the renal pelvis to the bladder. The peelaway sheath was then removed after indwelling a $16 \mathrm{~F}$ nephrostomy tube through the sheath.

Group 3 was treated with MPCNL with the aid of our patented system. The puncture point was in the 12th rib infracostal margin, between the posterior axillary line and scapula line. Zebra guidewire was inserted and fixed and dilatation of the percutaneous tract was performed serially, the same as the traditional MPCNL. A16Fr patented sheath (Figures 1 and 2) [4,5] was placed at the percutaneous access port. The small diameter nephroscope $(12.3 \mathrm{~F})$ was subsequently inserted through the sheath to observe stones. Intraoperatively, physical saline was used for irrigation. We used irrigation pump to pump the fluid in and the vacuum suctioning to get the fluid out. A holmium laser was used to break the stones and the vacuum suctioning device was used to clear gravel. After examination to find whether there were any residual stones inside the upper ureter, the renal pelvis, and the renal calyces, we antegradely indwelled a $6 \mathrm{~F}$ double-J pigtail stent from the renal pelvis to the bladder. The patented sheath was then removed after indwelling a $16 \mathrm{~F}$ nephrostomy tube through the sheath $[4,5]$.

All patients in groups 2 and 3 were examined to rule out residual stones by using KUB and B ultrasound 3 days postoperatively. If there were no residual stones, the nephrostomy tube was clamped for a day, and then was removed the next day if there were no symptoms such as flank pain or fever after the clamping.

All patients in 3 groups were rechecked for KUB and B ultrasonography 1 month postoperatively. The indwelling double J stent was removed 2 to 4 weeks postoperatively. All the patients were

Table 1. Comparison of general clinical data.

\begin{tabular}{|c|c|c|c|c|c|}
\hline \multicolumn{2}{|l|}{ Average age (year) } & \multirow{2}{*}{$\begin{array}{c}\text { URS } \\
46.4 \pm 15.1\end{array}$} & \multirow{2}{*}{$\begin{array}{c}\text { MPCNL } \\
45.1 \pm 15.2\end{array}$} & \multirow{2}{*}{$\begin{array}{c}\text { PMPCNL } \\
45.2 \pm 14.7\end{array}$} & \multirow{2}{*}{$\begin{array}{c}\mathbf{P} \\
0.58\end{array}$} \\
\hline & & & & & \\
\hline \multirow[t]{2}{*}{$\operatorname{Sex}(n)$} & Male & 54 & 56 & 53 & \multirow[t]{2}{*}{0.88} \\
\hline & Female & 37 & 35 & 38 & \\
\hline \multicolumn{2}{|l|}{ Stone burden $\left(\mathrm{mm}^{2}\right)$} & $134.2 \pm 83.3$ & $150.3 \pm 93.4$ & $158.7 \pm 96.8$ & 0.8 \\
\hline \multirow{2}{*}{$\begin{array}{l}\text { Number of cases with varying degrees } \\
\text { of hydronephrosis (n) }\end{array}$} & Moderate & 64 & 62 & 65 & \multirow[t]{2}{*}{0.87} \\
\hline & Severe & 27 & 29 & 26 & \\
\hline \multicolumn{2}{|l|}{ Number of Cases complicated by UTI (n) } & 13 & 15 & 15 & 0.68 \\
\hline
\end{tabular}




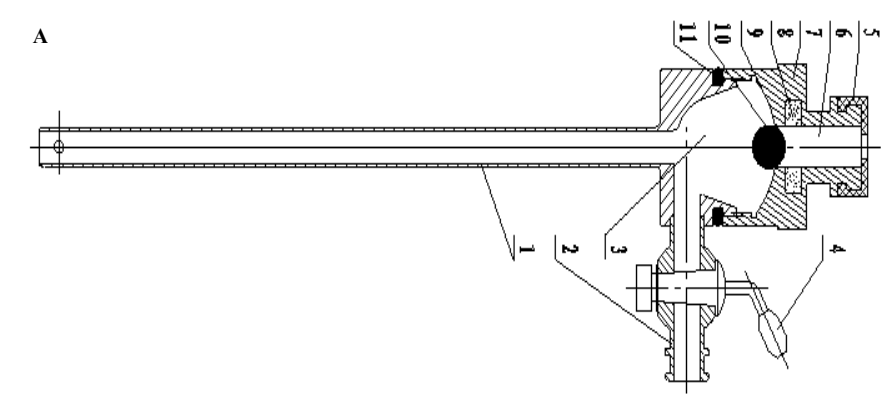

B

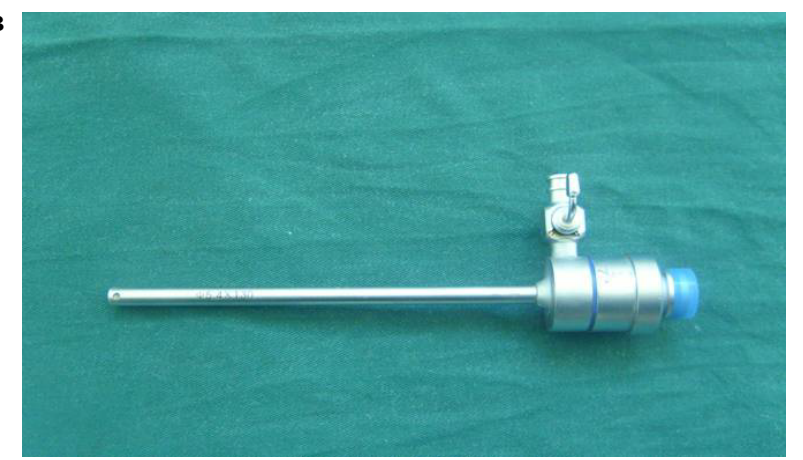

Figure 1. A. Parts of the patented sheath 1. Metal Sheath; 2. Side suction tube; 3. Funnelshaped internal cavity; 4. Valve of side suction tube; 5 . Sealed cap; 6 . Sealed working passage; 7. Sealed lid; 8. Permanent magnetic ring: 9. Hemisphere surface; 10. Magnetic steel ball; 11. Sealing soft ring. B. The patented sheath.

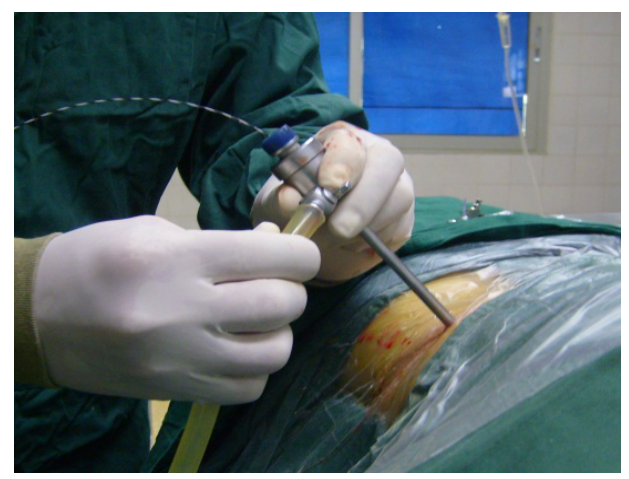

Figure 2. The patented sheath was connected to a vacuum device.

further followed up with KUB+IVU and ultrasonography for 3 to 12 months to investigate postoperative ureteral stricture and changes in the degree of hydronephrosis [4,5]. Due to the cost issue, we only performed postoperative CT for patients with radiolucent stones.

\section{Evaluation}

Operative time, amount of bleeding, need of blood transfusion, rate of postoperative fever, cost of treatment, rate of patients needing secondary SWL therapy, and stone clearance rate were recorded as data. Success treatment was defined as residual stones $<4 \mathrm{~mm}$ by KUB and B ultrasonography. The amount of intraoperative bleeding was calculated using a hydrogenated high iron hemoglobin method to measure the hemoglobin concentration in the suctioned fluid by the following formula: volume of blood loss $(\mathrm{ml})=[$ hemoglobin concentration in the suctioned fluid sample $(\mathrm{g} / \mathrm{L}) \mathrm{x}$ total volume of suctioned fluid $(\mathrm{ml})] /$ preoperative hemoglobin concentration $(\mathrm{g} / \mathrm{L})[4,5]$. The operating time in the MPCNL group was calculated from insertion of the percutaneous puncture needle to removal of the patented sheath and closure of its site. In the URS group, the operating time was calculated from entrance of the ureteroscope into the urethra until its withdrawal.

\section{Statistical methods}

The operative time, amount of bleeding and cost of treatment were compared by using Student's t test; rate of stone clearance, rate of postoperative fever and rate of cases needing postoperative SWL were compared by using Chi-Square test. Statistical significance was defined as $\mathrm{p}<0.05$.

\section{Results}

In the URS group, the operative time ranged from 20-90 min with an average value of $45.2 \pm 3.1 \mathrm{~min}$; the amount of intraoperative bleeding ranged from $6-25 \mathrm{ml}$ with an average value at $15.6 \pm 1.8 \mathrm{ml}$. There was no case that needed blood transfusion intraoperatively or postoperatively. There were 2 cases that were converted to MPCNL to clear stones due to the difficulty in inserting a ureteroscope. There was one case that was converted to open surgery to remove the stone due to significant ureteral injury during the endourological surgery. The stone clearance rate in this group was found to be $71.4 \% 3$ days postoperatively. There were 23 cases in this group who needed SWL therapy for residual stones. One month postoperatively, the stone clearance rate was found to be $89.0 \%$. There were 14 cases experiencing postoperative fever from $38.5-41^{\circ} \mathrm{C}$. Among these 14 patients, there was 1 case who suffered from infectious shock postoperatively (Grade IV by Clavien Classification), even though the patient was cured after anti-shock and anti-infection treatments. The average cost of treatment in this group for URS only was $1107.7 \pm 80.9$ US dollars. The average cost of treatment in this group for URS+SWL was 1252.1 \pm 96.9 US dollars. There were two cases in this group of postoperative ureteral stricture. One case was managed by endoureterotomy; the other case was managed by open primary ureteroureterostomy.

In the MPCNL group, the operative time ranged from 25 to 95 min with an average value at $43.4 \pm 2.5 \mathrm{~min}$; amount of intraoperative bleeding ranged from 45 to $200 \mathrm{ml}$ with an average value at $80.0 \pm 7.3$ $\mathrm{ml}$. There was no case that needed blood transfusion during the surgery. However, there were 2 cases who suffered from secondary bleeding postoperatively needing blood transfusion 200-600 ml. All patients were checked for potential residual stones 3 days postoperatively (Figure 3). The stone clearance rate was found to be $94 \%$. There were 3 cases in this group who needed SWL therapy for residual stones. One month postoperatively, the stone clearance rate was found to be $98 \%$. There were 9 cases in this group who experienced postoperative fever from $38.5-39.6^{\circ} \mathrm{C}$. However, the temperatures in these 5 patients returned to normal after anti-inflammatory treatment. No serious complications such as organ injury in the abdomen, pneumothorax, infectious shock, or hemorrhagic shock occurred. The average cost of treatment in this group was 10123.1 $\pm 1063.7 \mathrm{RMB}$. There was one case in this group of postoperative ureteral stricture, which was managed by endoureterotomy.

In the PMPCNL group, the operative time ranged from 15 to 75 min with an average value at $27.4 \pm 2.3 \mathrm{~min}$; amount of intraoperative bleeding ranged from 25 to $100 \mathrm{ml}$ with an average value at $40.2 \pm 5.3$ $\mathrm{ml}$. There was no case that needed blood transfusion during the surgery. However, there were 2 cases who suffered from secondary bleeding postoperatively needing blood transfusion $200-400 \mathrm{ml}$. All patients were checked for potential residual stones 3 days postoperatively (Figure 3). The stone clearance rate was found to be $97.8 \%$. One month postoperatively, the stone clearance rate was found to be $100 \%$. There 
were 5 cases in this group who experienced postoperative fever from $38.5-39.6^{\circ} \mathrm{C}$. However, the temperatures in these 5 patients returned to normal after anti-inflammatory treatment. No serious complications such as organ injury in the abdomen, pneumothorax, infectious shock, or hemorrhagic shock occurred. The average cost of treatment in this group was $1592.1 \pm 166.4$ US dollars. There was no case of postoperative ureteral stricture identified during the follow-up period.

MPCNL and PMPCNL were superior to URS to a statistically significant degree in treating upper ureteral impacted stones with regards to operative time, rate of postoperative fever, need for postoperative SWL therapy to treat residual stone, and stone clearance rate. However, there was a significantly higher amount of bleeding and higher cost of treatment in the MPCNL and PMPCNL groups. PMPCNL was superior to MPCNL with regards to operative time, rate of postoperative fever, stone clearance rate by one surgery, and incidence of ureteral stricture (Table 2).

A

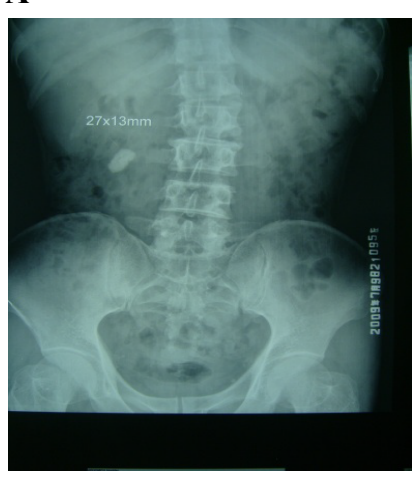

B

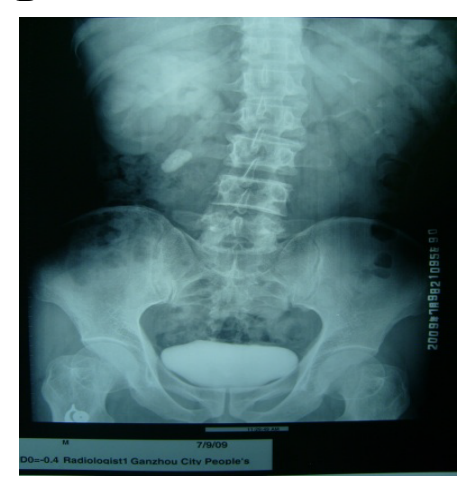

C

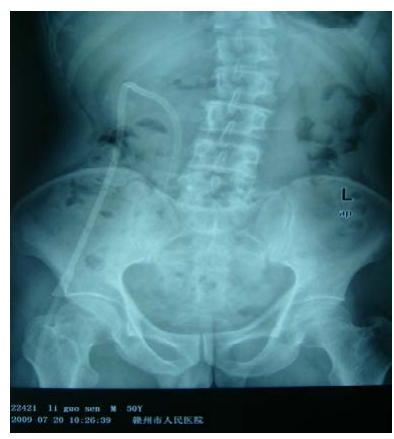

Figure 3. Single-channel lithotripsy was successfully performed in a 50-year-old male with a right upper ureteral stone within 20 minutes. Preoperative and postoperative images are presented. A. Preoperative KUB; B. Preoperative IVU; C: Postoperative KUB.

\section{Discussion}

For upper ureteral stone which cannot be micturated out by the patient spontaneously after conservative therapies, the main treatment options are SWL, ureteroscopic lithotripsy, and PCNL. In rare circumstances there is a need for open surgery or laparoscopic surgery [6]. SWL is generally used as the first choice for ureteral stones that are not impacted. For impacted ureteral stones, or ureteral stones in patients with stricture or distortion in the urinary tract below the stones, or ureteral stones complicated by ipsilateral kidney stones or serious renal insufficiency, the outcome of SWL therapy is not good [3].

Ureteroscopy has become one of the main options in treating ureteral stones due to its advantages including minimal invasiveness and the ability to treat bilateral ureteral stones conveniently through natural anatomic tracts. However, in the present study stone clearance was significantly poorer than PMPCNL when URS was used to treat upper ureteral stones. The main cause of this poorer performance may include the following factors: 1) the stone or gravels are easily move to the renal pelvis or renal calyces during the operation; 2 ) it can be difficult to insert ureteroscope if there is significant stricture or distortion in the ureteral segments below the ureteral stone; 3 ) it can be difficult in perform lithotripsy when the surgical field is obscured by bleeding from granular tissue surrounding the stone. In addition, saline infusion under a high pressure during ureteroscopic lithotripsy frequently leads to high fever and flank pain [5].

When treating impacted upper ureteral stones using a Holmium laser, the chance of reflux of the stones to the renal pelvis or renal calyces is relatively low because of the relative fixation of the impacted stone to the ureter. However, we still saw stone or large gravel reflux to the kidney in $25.3 \%$ (23/91) cases, which meant that secondary SWL therapy was needed. There were 2 cases that had to be converted to PMPCNL to clear stones due to the difficulty in inserting a ureteroscope and one case that was converted to open surgery to remove the stone due to significant ureteral injury during the endourological surgery. There were $13.2 \%$ cases that experienced postoperative fever including one case that suffered from infectious shock. Therefore, when using URL in treating upper ureteral stones, it is important not to forcibly insert the ureteroscope when there is significant difficulty, in order to avoid ureteral injury. Even though we need to keep the surgical field as clear as possible, we should not infuse the patient with saline continuously under high pressure, to avoid high pressure inside the renal pelvis and the possibility of stone or gravel reflux into the kidney. We suggested that the time and pressure of the infusion should be controlled, and we should infuse and drain alternately, especially in cases with preoperative urinary tract infection and fever, in order to avoid septicemia. The main goal of URL is to break the stone into small

Table 2. Comparison for intraoperative and postoperative clinical data.

\begin{tabular}{|l|c|c|}
\hline \multicolumn{1}{|c|}{ PMPCNL } & MPCNL \\
\hline Patient number (n) & 91 & 91 \\
\hline Average operative time(min) & $45.2 \pm 3.1$ & $43.4 \pm 2.5$ \\
\hline Average amount of bleeding (ml) & $15.6 \pm 1.8$ & $80.0 \pm 7.3 \Delta$ \\
\hline Rate of Postoperative fever (\%) & $14(15.4)$ & $9(9.9) \Delta$ \\
\hline Number of cases needing postoperative SWL therapy (n) & 23 & $3 \Delta$ \\
\hline Cost of treatment (Chinese Yuan) & $7037.2 \pm 514.3$ & $10123.1 \pm 1063.7 \Delta$ \\
\hline Stone clearance rate (3 days postoperatively) & $71.4 \%(65 / 91)$ & $94 \%(86 / 91) \Delta$ \\
\hline Stone clearance rate (1 month postoperatively) & $89 \%(81 / 91)$ & $10115.1 \pm 1057.3 *$ \\
\hline Number of cases with postoperative ureteral stenosis (n) & 2 & $97 \%(89 / 91) * *$ \\
\hline
\end{tabular}

MPCNL group compared with URL group, $\mathrm{P}<0.05 ;$; PMPCNL group compared with URL group, $\mathrm{P}<0.05$, but when compared with MPCNL, $\mathrm{P}>0.05$; **:PMPCNL group compared with URL group and MPCNL group, $\mathrm{P}<0.05$

$\Delta$ : MPCNL vs. URL, $\mathrm{P}<0.05 ; *: \mathrm{PMPCNL} v$ s. URL， $\mathrm{P}<0.05$, but PMPCNL vs. MPCNL, $\mathrm{P}>0.05 ; * *: \mathrm{PMPCNL} v s . \mathrm{URL}, \mathrm{P}<0.05$, and $\mathrm{PMPCNL} v s . \mathrm{MPCNL}, \mathrm{P}<0.05$ 

impacted upper ureteral stones

pieces. It is not advisable to retrieve every small piece of gravel out of the ureter manually, because of the risk of ureteral injury. We suggest that the laser lithotripsy be done by using a tiny optic fiber cord under a low energy setting to bite the stone beginning from the edge. This was found to be able to increase the rate of success in stone-breaking.

In 1996, Kumar et al. reported that the stone clearance rate was as high as $86 \%$ by using standard PCNL in treating upper ureteral stones and thought the outcome was much better than SWL therapy [7]. However, in standard PCNL, due to the larger size of the percutaneous tract, there is larger amount of intraoperative bleeding and a higher rate of postoperative complications, compared with MPCNL. Also due to the larger sized nephroscope used in standard PCNL, which leads to greater difficulty in accessing the ureter. In most circumstances, standard PCNL can only deal with ureteral stones above the level of L3 [8]. MPCNL has been extensively performed over recent years, Jackman and Lahme et al. concluded that MPCNL had significant advantages, including fewer injuries, greater ease of use, less intraoperative bleeding, and fewer major complications $[9,10]$.

Application of traditional MPCNL using a peel-away sheath is an effective way to treat upper urinary tract calculi. However, this technique clears stones away mainly through high-pressure perfusion or the use of pliers to remove stones manually, could therefore lead to significant complications. Another major disadvantage is that it lengthens the duration of the surgery.

In order to increase stone clearance rate and reduce complications, we have designed a PMPCNL with suctioning ability. Our percutaneous tract was established the same way as a traditional MPCNL; however, we used a patented suctioning stone-breaking and clearance sheath to replace the peel-away sheath. The patented sheath was also connected to a vacuum device to increase aspiration capability [4]. Suctioning under negative pressure can easily clear the hydrops in the collecting system. In the meantime, we did not raise the abdomen to fix the kidney so the movement range of the kidney was increased. The stone was mainly broken by a Holmium laser in a cutting fashion. All the gravel less than $5 \mathrm{~mm}$ could be sucked away during stone-breaking [4]. Low negative pressure in the renal pelvis guaranteed that the gravels would not easily move downward in the ureter, nor would they be refluxed to the kidney. Impacted ureteral gravels surrounded by the ureteral polyps could be moved by the patented sheath and then be suctioned out. Thus the efficacy of gravel clearance was significantly increased, while the rates of retrograde infection and fluid extravasations were significantly decreased. In our PMPCNL group, the stone clearance rate by one surgery was $100 \%$. There were no serious complications in this group. The stone clearance rate was significantly higher while the rate of complications was less than in the group who had URS treatment. The outcome of treatment using PMPCNL was also superior to that in other stone treatment centers in China where traditional MPCNL was performed, with a shorter operative time and fewer complications [5, 11,12 . Even though there are so many advantages to use PMPCNL, we still need to keep the balance of irrigation and suction under negative pressure, and avoid high pressure in the renal pelvis, especially when the lateral hole is occluded by gravel.

In conclusion, there are different advantages and disadvantages in using PMPCNL, MPCNL or URS in treating impacted upper ureteral stones. In the case of URS there is no need to establish an artificial operative pathway and less injury. However, there are disadvantages to it, including a low stone clearance rate, a longer time needed to clear the stone, and a higher need for postoperative SWL therapy.
There is certain injury when using PMPCNL or MPCNL to treat upper ureteral impacted stones. However, there was no increased rate of complications generally, with great advantages, including high efficacy, great safety, high stone clearance rate, and a shorter operative time. PMPCNL was superior to MPCNL with regards to operative time, rate of postoperative fever, and stone clearance rate by one surgery. We think PMPCNL with the aid of the patented system could be the first choice in treating complicated impacted upper ureteral stones above the level of L4, warranting an extensive use.

\section{Conflicts of interest}

None declared.

\section{Consent}

Written informed consent was obtained from the patients for publication of this report and any accompanying images. Copies of the written consent are available for review by the Editor of the journal.

\section{Ethical standard}

Ethical and regulatory approvals were sought and obtained from all the 4 participating hospitals.

\section{Author contribution}

Protocol/project development: Song, Yang, Liu, Pan, Chen, Ye Data collection or management: Luo, Yang, Liao, Xie, Qiu, Deng, Zhu, Fan, Peng, Qin, Liu, Pan, Chen Data analysis: Xie, Yang, Luo, Liao Manuscript writing/editing: Xie, Yang, Song, Liao.

\section{Acknowledgements}

This work was supported by major scientific and technological project funds of Jiangxi Provincial Health Department, Jiangxi, China (20094015) to L.M.S.

\section{References}

1. Rops AL, van der Vlag J, Lensen JF, Wijnhoven TJ, van den Heuvel LP, et al. (2004) Heparan sulfate proteoglycans in glomerular inflammation. Kidney Int 65: 768-785. [Crossref]

2. Raats CJ, Van Den Born J, Berden JH (2000) Glomerular heparan sulfate alterations mechanisms and relevance for proteinuria. Kidney Int 57: 385-400. [Crossref]

3. Litwack ED, Ivins JK, Kumbasar A, Paine-Saunders S, Stipp CS, et al. (1998) Expression of the heparan sulfate proteoglycan glypican-1 in the developing rodent. Dev Dyn 211: 72-87. [Crossref]

4. Saunders S, Paine-Saunders S, Lander AD (1997) Expression of the cell surface proteoglycan glypican-5 is developmentally regulated in kidney, limb, and brain. Dev Biol 190: 78-93. [Crossref]

5. Grisaru S, Cano-Gauci D, Tee J, Filmus J, Rosenblum ND (2001) Glypican-3 modulates BMP- and FGF-mediated effects during renal branching morphogenesis. Dev Biol 231: 31-46. [Crossref]

6. Okamoto K, Tokunaga K, Doi K, Fujita T, Suzuki H, et al. (2011) Common variation in GPC5 is associated with acquired nephrotic syndrome. Nat Genet 43: 459-463.

7. Filmus J, Capurro M, Rast J (2008) Glypicans. Genome Biol 9: 224. [Crossref]

8. Gupta M, Brand M. (2013) Identification and expression analysis of zebrafish glypicans during embryonic development. PLoS One 8: e80824. [Crossref]

9. Hanke N, Staggs L, Schroder P, Litteral J, Fleig S, et al. (2013) "Zebrafishing" for novel genes relevant to the glomerular filtration barrier. Biomed Res Int 2013: 658270. [Crossref]

10. Xie J, Farage E, Sugimoto M, Anand-Apte B (2010) A novel transgenic zebrafish model for blood-brain and blood-retinal barrier development. BMC Dev Biol 10: 76. [Crossref]

11. Bollig F, Mehringer R, Perner B, Hartung C, Schäfer M, et al. (2006) Identification 
and comparative expression analysis of a second wt1 gene in zebrafish. Dev Dyn 235: 554-561. [Crossref]

12. Hentschel DM, Mengel M, Boehme L, Liebsch F, Albertin C, et al. (2007) Rapid screening of glomerular slit diaphragm integrity in larval zebrafish. Am J Physiol Renal Physiol 293: F1746-1750. [Crossref]

13. Kirsch T, Kaufeld J, Korstanje R, Hentschel DM, Staggs L, et al. (2013) Knockdown of thehypertension-associated gene NOSTRIN alters glomerular barrier function in zebrafish (Danio rerio). Hypertension 62: 726- 730. [Crossref]

14. Drummond IA, Majumdar A, Hentschel H, Elger M, Solnica-Krezel L, et al. (1998) Early development of the zebrafish pronephros and analysis of mutations affecting pronephric function. Development 125: 4655-1467. [Crossref]

15. Tryggvason K, Patrakka J, Wartiovaara J (2006) Hereditary proteinuria syndromes and mechanisms of proteinuria. $N$ Engl J Med 354: 1387-1401. [Crossref]

16. Gengrinovitch S, Berman B, David G, Witte L, Neufeld G, et al. (1999) Glypican-1 is a VEGF165 binding proteoglycan that acts as an extracellular chaperone for VEGF165. J Biol Chem 274: 10816-10822. [Crossref]

17. Zeng Y, Tarbell JM2 (2014) The adaptive remodeling of endothelial glycocalyx in response to fluid shear stress. PLoS One 9: e86249. [Crossref]

18. Ebong EE, Lopez-Quintero SV, Rizzo V, Spray DC, Tarbell JM (2014) Shear-induced endothelial NOS activation and remodeling via heparan sulfate, glypican-1, and syndecan-1. Integr Biol (Camb) 6: 338-347. [Crossref]
19. Stein JM (1975) The effect of adrenaline and of alpha- and beta-adrenergic blocking agents on ATP concentration and on incorporation of 32Pi into ATP in rat fat cells. Biochem Pharmacol 24: 1659-1662. [Crossref]

20. Rosenberg RD, Shworak NW, Liu J, Schwartz JJ, Zhang L. (1997) Heparan sulfate proteoglycans of the cardiovascular system. Specific structures emerge but how is synthesis regulated? J Clin Invest 100: S67-75. [Crossref]

21. Mertens G, Cassiman JJ, Van den Berghe H, Vermylen J, David G. (1992) Cell surface heparan sulfate proteoglycans from human vascular endothelial cells. Core protein characterization and antithrombin III binding properties. J Biol Chem 267: 20435 20443. [Crossref]

22. Karumanchi SA, Jha V, Ramchandran R, Karihaloo A, Tsiokas L, et al. (2001) Cell surface glypicans are low-affinity endostatin receptors. Mol Cell 7: 811-822. [Crossref]

23. Doi K, Noiri E, Fujita T (2010) Role of vascular endothelial growth factor in kidney disease. Curr Vasc Pharmacol 8: 122-128. [Crossref]

24. Ray PE, Liu XH, Xu L, Rakusan T. (1999) Basic fibroblast growth factor in HIVassociated hemolytic uremic syndrome. Pediatr Nephrol 13: 586-593. [Crossref]

25. Floege J, Kriz W, Schulze M, Susani M, Kerjaschki D, et al. (1995) Basic fibroblast growth factor augments podocyte injury and induces glomerulosclerosis in rats with experimental membranous nephropathy. J Clin Invest 96: 2809-2819.

26. Kriz W, Hahnel B, Rosener S, Elger M. (1995) Long-term treatment of rats with FGF-2 results in focal segmental glomerulosclerosis. Kidney Int 48: 1435-1450.

Copyright: $\odot 2017$ Yang Z. This is an open-access article distributed under the terms of the Creative Commons Attribution License, which permits unrestricted use, distribution, and reproduction in any medium, provided the original author and source are credited. 